\section{Elastic or inelastic compression? Reported evidence from clinical trials}

\section{Mieke Flour \\ University Hospital Leuven, Belgium}

\section{Abstract}

Evidence for compression therapy found in literature mainly comes from clinical studies, preferably randomized controlled trials (RCTs) and systematic reviews (SR), which are often complemented by research data, expert opinion or by data from technology assessment or regularization documents. Differences between materials/methods/intervention in clinical trials can be partly explained by variability in focus, or due to country specific issues. Results from RCTs and SRs, and the interpretation of these results may vary depending on definitions used and the adequacy of data. In the first place, the baseline comparability of study groups depends very much on the accuracy of the diagnosis. Secondly, results will very much depend on the intervention used, whether compression is used alone, or whether it is part of a more complex management like decongestive treatment including other physical methods, surgery, or pharmacological treatment. A third consideration relates to the outcome parameters, the methods used to measure them, and the length of follow-up. Properties of compression materials have been redefined and standardized, and new insights in the physiological effects of compression treatment have shaken existing myths and dogmas in this field. RCTs using out-dated definitions and classifications of materials have led to systematic reviews and recommendations based on the same misunderstanding; it is left to the alert reader to interpret their results with caution.

\section{Introduction}

Evidence for compression therapy found in literature mainly comes from clinical studies, preferably randomized controlled trials (RCTs) and systematic reviews (SR), which are often complemented by research data, expert opinion or by data from technology assessment or regularization documents [European Committee for Standardization (CEN), ReichsAusschuss für Lieferbedingungen Gütezeichengemeinschaft (RAL-GZG), British Standards Institution (BSI) ]. ${ }^{1-3}$
Grading and definition of the level of the selected evidence vary between publications, and this is usually described in the introduction of the manuscript. Either there will be some objective ranking of the quality/reliability of trials and evidence, or the recommendations combine objective ranking of the evidence with other considerations for practice, like the GRADE tool introduced by the American College of Chest Physicians. ${ }^{4}$

There aren't too many new relevant good quality RCTs each year, so there will not be too much difference between the source documents for systematic reviews, and thus most recommendation documents on compression therapy look very much alike indeed.

\section{Variability in trials' setup}

Differences between materials/methods/ intervention in clinical trials can be partly explained by variability in focus, or due to country specific issues.

Focus may be differently accentuated $e . g$. depending on authorship and target users groups (nurses, versus medical specialties, versus true multidisciplinary groups including patients' representatives). Also, the scope may vary, depending on whether the intervention is purely conservative (compression treatment, education, etc.) versus that the consensus includes additional recommendations on medical/surgical interventions for etiological management and follow-up of the underlying disease (venous, lymphatic, thrombosis, etc.). Of course, the scope will also depend on the specific selected indication or goal setting: the trial or his outcomes may be aiming at getting reimbursement from health care institutions (like those from the Haute Autorité de santé in France, the Dutch Institute for Healthcare Improvement in the Netherlands), or aiming at setting educational endpoints, or it is meant for implementation of the uniform application of materials and techniques throughout the country (like in the Netherlands, or the 4-layer bandaging in the UK). Country specific issues may be the selection of bandages/stocking types according to availability or local preferences (stockings preferred above bandages in France? inelastic bandages preferred in the older guidelines in some European countries). National guidelines/recommendations on compression treatment in specific indications exist in countries like France, the Netherlands, UK, Ireland, Italy, Germany, Canada, Australia, New Zealand, Belgium, and many others.

National and International Societies have issued consensus documents or best practice documents regarding compression therapy, and this will most probably influence the choice of intervention by investigators.
Correspondence: Mieke Flour, Schoonzichtlaan 43, B-3020 Herent, Belgium. Tel. +32.478.566780. E-mail: mie.flour@skynet.be

Key words: compression treatment, elastic, inelastic, evidence, clinical trials.

Conference presentation: part of this paper was presented at the International Compression Club (ICC) Meeting on Stiffness of Compression Devices, 2012 May 25, Vienna, Austria (http://www.icc-compressionclub.com/).

Conflict of interests: the author reports no potential conflict of interests.

Received for publication: 14 November 2012

Revision received: 2 January 2013.

Accepted for publication: 21 January 2013.

This work is licensed under a Creative Commons Attribution 3.0 License (by-nc 3.0).

(C) Copyright M. Flour, 2013

Licensee PAGEPress, Italy

Veins and Lymphatics 2013; 2:e7

doi:10.4081/vl.2013.e?

\section{Variability in trial outcomes and recommendations}

Results from RCTs and SRs, and the interpretation of these results may vary depending on definitions used and the adequacy of data. In the first place, the baseline comparability of study groups depends very much on the accuracy of the diagnosis. In still too many trials and reviews the venous ulcer etiology is based on a normal ankle brachial pressure index in a patient with a leg ulcer clinically compatible with a venous ulcer. Not only can the diagnosis (based only on clinical examination) be erroneous, it can underestimate the severity and extent of the problem and any relevant co-morbidity. The accuracy of a venous etiological diagnosis increases with the addition of imaging and invasive testing in chronic venous disorders. The American Venous Forum recommends duplex scanning as the first diagnostic test to all patients with suspected chronic venous obstruction or valvular incompetence.

Secondly, results will very much depend on the intervention used, be it compression alone (on top of dressing choice), or complex decongestive treatment including other physical methods [physical therapy, intermittent pneumatic compression (IPC)], or a combination of treatments including surgery, or pharmacological treatment. In the Materials and Methods paragraph of published trials and studies, description of the type of compression treatment should clearly state specific details, such as: what is the definition and classification 
used for compression materials (bandages, stockings), is the applied pressure or stiffness measured in vivo, is compression strength described in (country specific) compression classes or in $\mathrm{mmHg}$, are the bandages and stockings named and described so the reader can agree or not with the label used for compression materials (e.g. definitions like in the BSI, or definitions like inelastic, elastic, short stretch, long stretch, superposition of layers, etc.). A third consideration relates to the outcome parameters, the methods used to measure them, and the length of follow-up.

Interpretation of the results and the recommendations issued from it, are very much dependent on the above listed issues and insights, which themselves have been the topic of several recent consensus documents. ${ }^{5-7}$

\section{Evidence for the effectiveness of compression therapy}

A comprehensive review of evidence regarding effectiveness of compression therapy in several venous indications following the Clinical, Etiology, Anatomy, Pathophysiology (CEAP) classification and scoring system, and in lymphoedema can be found in the Consensus document published by the International Compression Club (ICC) in
2008. ${ }^{8}$ These indications are listed in Table 1 and include clinical stages of venous disease, treatment following phlebological interventions, venous thrombosis and lymphoedema.

The compression devices used in the trials for these indications include bandages, stockings, ortheses [like Circaid ${ }^{\mathrm{TM}}$ (San Diego, CA, USA), tubular elastic cotton sleeves like Tubigrip $^{\mathrm{TM}}$ (Mölnycke Health Care, Gothenburg, Sweden), Tubulcus ${ }^{\mathrm{TM}}$ (INNOTHERA CH S.A. Service Zentrum Europa, Saint Blaise, Switzerland)], and IPC. Not all indications have been adequately studied regarding effectiveness of compression treatment, partially due to the fact that measurement outcomes are not always easily defined or assessed, and that they will be dictated by the indication at study. In this Table the references get a GRADE-label for the recommendation (e.g. 1B, 1A), and the insertion of weighted/graded references under a specific column head is deducted from the original classes mentioned on the respective documents. Also, the pressure values are rounded to simplified ranges. Reason for this is the known discordance between several country specific classifications of pressure range for stockings, and variations in definition of expected pressure under bandages when applied according to the manufacturers' instructions. This Table does not distinguish between elastic or inelastic materials. In most trials, measurement of the delivered pressure was not measured in vivo, neither was the stiffness index. Duration of follow-up in RCTs is understandably limited, variable, and not always representative for the selected disease progression; thus in some indications the deducted recommendation of duration of treatment are decided by consensus or by expert opinion. An example to illustrate this fact is compression therapy in venous disease $\mathrm{C} 4 \mathrm{a}$, C4b, C5. Experimental data exist, but Clinical Trial data are lacking; Class III medical compression stockings said to deliver $30-40 \mathrm{mmHg}$ have been shown to reduce the area of lipodermatosclerosis (LDS) in patients with healed venous ulcers. ${ }^{9}$ Accordingly it is also considered to improve areas of atrophie blanche and to reduce the edema and induration in the leg associated with these conditions. There are experimental data supporting effectiveness of distinct levels of compression regarding different aspects of LDS: reduction of edema, eczema, iron deposition, area of LDS, inflammation and pain, but no RCT's have been found. Clinical trials evaluating compression treatment specifically in lipodermatosclerosis are rare, presumably due to the many possible outcome parameters to choose from, of which validation is not established for the specific indication. Progression to ulceration, and prevention of this by the specific compression device is difficult to predict and so it is hard to calculate the power needed to demonstrate effectiveness.

Table 1. Indications for compression treatment. Reported efficacy of compression therapy stockings, bandages and intermittent pneumatic compression by randomized controlled trials and meta-analyses in patients with chronic venous disorders (Clinical, Etiology, Anatomy, Pathophysiology classification), venous thromboembolism and lymphedema. Only strong grades of recommendations are indicated: $1 \mathrm{~A}$ and $1 \mathrm{~B}$. Adapted from Partsch et al., 2008. ${ }^{8}$

$\begin{array}{lcc}\text { Indications CEAP } & \text { Compression stockings } & \text { Bandages } \\ & 10-20 \mathrm{mmHg} & \text { Compression pressure in } \mathrm{mmHg} \\ & 20-30 \mathrm{mmHg} & 30-40 \mathrm{mmHg}\end{array}$

$\mathrm{COs}, \mathrm{Cls}$

$1 \mathrm{~B}$

$\mathrm{Cl}$ after sclero

$1 \mathrm{~B}$

$\mathrm{C} 2 \mathrm{a}, \mathrm{s}$

$\begin{array}{lll}\text { C2s pregnancy } & 1 \mathrm{~B} & 1 \mathrm{~B} \\ \mathrm{C} 3 \text { prevention } & 1 \mathrm{~B} & -\end{array}$

C3 therapy

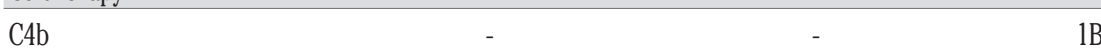

$\mathrm{C} 5$

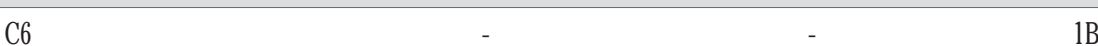

After procedures

VTE prevention therapy

\section{B}

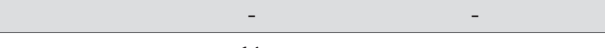

$1 \mathrm{~A}$

B $1 \mathrm{~B}$

$1 \mathrm{~B}$

PTS prevention therapy 
Indications define the measured outcomes in clinical trials for compression treatment in venous disease, and unfortunately, there is hardly any objective standard assessment method for most outcome parameters in the clinical classes preceding an ulcer. This is one of the reasons why clinical trials are hard to find in these indications. Another explanation is that clinical progression and thus effectiveness of compression is difficult to predict during the relatively short time laps of a trial.

In the early stages of venous disease, like $\mathrm{Cl}$ and $\mathrm{C} 2$, subjective symptoms are not always present, they are not always specific nor diagnostic, and clinical progression is unlikely to be influenced by compression treatment of a trial duration. Side-effects of compression may be considered as secondary outcome, but then these would not be correlated to the clinical stage of disease. For venous edema C3, symptoms and signs may be measured although there are many different ways to do this. Several trials have demonstrated edema reduction with the use of bandages and stockings, but have not been withheld in the abovementioned publication (Table 1). ${ }^{8}$ As mentioned in Table 1, compression treatment has been positively evaluated in C5 for the prevention of ulcer recurrence, as compared to surgery. Most clinical trials have been performed on C6, venous ulceration, evaluating wound healing as a primary outcome parameter. This is an objectively measurable outcome, and compression materials or application methods can be compared for effectiveness. Prevention of recurrence has been studied as well, as mentioned earlier. Disease specific quality of life issues have also been evaluated, in contrast to resolution of skin changes for which no clinical trial could be found. The problem here is that dosimetry and characteristics of the compression therapy are debatable, due to outdated or confusing definitions and classification of bandages or stockings. Indeed, new insights in the physiological effects of compression treatment and updated consensus documents invite us to re-interpret older trial data. This fact relates to the intervention itself. Another consideration is that methodology of selected clinical trials fulfils historical quality requirements. But expectations and quality criteria become more stringent over the years (the rules of the game change while playing). Also, good trial-methods do not necessarily guarantee a correct diagnosis or scoring/grading of the clinical disease at study. This will of course apply to the systematic reviews or guidelines and consensus documents derived from these trials. That fact can be named the inherent tragedy of initiatives like the Cochrane database and many other institutions gathering evidence and knowledge: a RCT perfectly meeting today's strict requirements may be outdated and rejected in a later systematic review as soon as new insights change the rules.

\section{New insights in compression treatment}

Interpretation of the trial results and the recommendations issued from it, are very much dependent on the above listed issues and insights, which themselves have been the topic of several recent consensus documents. ${ }^{5-7}$

In this issue several other contributions debate the stiffness or elasticity of compression materials and the measured physiological effects on the treated limb.

Properties of compression bandages have been updated in a publication reviewing the practical aspects and definitions. ${ }^{6}$ In that article the acronym PLACE is proposed to summarize the essential aspects that impact on the pressure and stiffness of compression materials. These are the sub-bandage pressure range measured at the gaiter area, the number of layers (and the way they overlap), the several components of the bandage each with its own function (like padding, protection, retention, compression), and the elastic properties or behavior of the assembled bandage. This is why pressure and stiffness must (also) be measured in vivo, on the treated limb.

Appropriate selection and use of these four properties will define the compression treatment characteristics and effectiveness in the several indications. The term dosimetry of compression pressure has been proposed to describe this.

In past clinical trials on compression treatment for venous and lymphatic disease, little is known about dosimetry of the applied compression, for how long and at what level it was or should be applied to yield the described results. The different effects of elastic versus inelastic or short-stretch compression are also little understood without considering the principle of stiffness and the resulting dynamic behavior of the compression device, which is rarely discussed in most selected trials and reviews.

RCTs using out-dated definitions and classifications of materials have led to systematic reviews and recommendations based on the same misunderstanding; it is left to the alert reader to interpret their results with caution. The pressure-range classifications of bandages and stockings are country specific, and so are the brands and trade names. There is yet no universally accepted standard terminology or classification, application technique or methodology to apply compression treatment. The number of publications is steadily growing with research data, but there is no universal estimation of pressure- values in vivo (which is dependent of the material used, the care giver, and the patient), and therefore there is no consensus yet on the required pressure, stiffness or compression technique to obtain results in specific indications.

The abovementioned considerations may provide part of the explanation for the wide variability in the materials and methods section of the several RCTs. Sound description of the dosimetry must include components, duration, pressure, layers, elasticity, stiffness, all aspects for which internationally accepted definitions are recently published, but not implemented yet, and thus not used in older trials. There is an impressive choice of compression materials and techniques like bandages, stockings, ortheses, intermittent pneumatic compression devices, and combinations of all these. As for the duration of compression therapy, this may be sustained, with or without changes during the day, or it may change over time, possibly in a cross-over study design. Of course, the applied pressure values or compression classes must be explicitly mentioned, referring to the methods for the in vivo assessment of pressure and stiffness. Blinding cannot be done for the application, but must be used for outcome assessment.

Practical problems abound when considering clinical trials on compression treatment for chronic venous leg ulcers: even if investigators do manage to agree between centers on a standardized protocol regarding materials and techniques, there is still a wide variation in the limbs under study, and there are many possible outcome parameters to test, which are not always under control or not always objectively measurable. Due to the variability of limb morphology, mobility, underlying (co-) morbidities and ulcer etiology, response to treatment will remain an individual characteristic confounding baseline comparability of studied subjects.

Nevertheless, as stated in almost all guidelines and systematic reviews, it is probably true to conclude that to heal a venous leg ulcer (C6), management that includes compression is more effective than without compression, that higher pressure (stiffness?) is more effective than low pressure values, and that compression should stay in place as long as possible. It is unclear if this means sustained pressure by elastic systems or pressure peaks under inelastic bandaging systems or stiff stockings. Some reviewers also recommend applying the highest pressure tolerated by the patient, although this may negatively influence compliance/adherence to treatment, and secondly, this statement has been refuted by recent trials in secondary lymphedema, which strongly suggest that there is a window of optimal pressure values for achieving edema reduction. The same may be true for ulcer healing, effect on skin changes, inflammation, or subjective symptoms. ${ }^{10}$ 


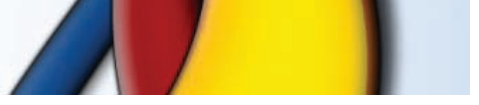

\section{Conclusions}

In order to compare the effectiveness of compression systems and materials, modern terminology (for which consensus exists) shall be used to describe the materials applied, and objective measurement of the dosimetry (pressure/stiffness/dynamic behavior/duration) is an added value in future trials and systematic reviews. Recommendations to guide clinicians and researchers hereby have been reported by consensus working groups. ${ }^{11}$ The methodological validity and quality of selected previous RCTs remains, but we may have to re-interpret the results in the light of new insights which have challenged myths and dogmas ${ }^{10}$ concerning hemodynamic effects of elastic and inelastic compression treatment, and concerning pressure and stiffness, the characteristics of the final compression system more than those of the individual components used.

\section{References}

1. European Committee for Standardization (CEN). Medical compression hosiery. ENV 12718:2001 E, August 2001. Brussels:
European Committee for Standardization; 2001. Available from: www.cenorm.be/ catweb/; www.cen.eu

2. RAL-GZ 387. Deutsches Institut für Gütesicherung und Kennzeichnung Medizinische Kompressionsstrümpfe RALGZ 387. Neufassung Sept 2000. Berlin: Beuth-Verlag; 2000. Updated in: RAL GZ 387 for compression stockings: Gütezeichengemeinschaft Medizinische Kompressionsstrümpfe; January 2008. Available from: http://www.gzg-kompressionsstruempfe.de/

3. British Standards Institution. Specification for the elastic properties of flat, non- adhesive, extensible fabric bandages. BS 7505:1995. Updated: January 2011. Available from: http://www.standardscentre.co.uk/bs/BS-7505-1995/?s=1

4. Guyatt G, Gutterman D, Baumann MH, et al. Grading strength of recommendations and quality of evidence in clinical guidelines. report from an American College of Chest Physicians Task Force. CHEST 2006;129:174-81.

5. Clark M. Compression bandages: principles and definitions. In: European Wound Management Association. Understanding compression therapy; position document of the European Wound Management
Association. Frederiksberg: Medical Educational Partnership Ltd; 2003. Available from: http://www.ewma.org

6. Partsch H, Clark M, Mosti G, et al. Classification of compression bandages: practical aspects. Dermatol Surg 2008; 34:600-9.

7. Partsch H, Clark M, Bassez S, et al. Measurements of interface pressure and stiffness. Dermatol Surg 2006;32:224-33.

8. Partsch H, Flour M, Coleridge Smith P, et al. Indications for compression therapy in venous and lymphatic disease. Consensus based on experimental data and scientific evidence; Under the auspices of the IUP. Int Angiol 2008;27:193-219.

9. Vandongen YK, Stacey MC. Graduated compression elastic stockings reduce lipodermatosclerosis and ulcer recurrence. Phlebology 2000;15:33-7.

10. Flour M, Clark M, Partsch H, et al. Dogmas and controversies in compression therapy: Report of an International Compression Club (ICC) meeting, Brussels, May 2011. Int Wound $\mathrm{J}$ 2012. [Epub Ahead of Print].

11. Rabe E, Partsch $H$, Jünger $M$, et al. Guidelines for clinical studies with compression devices in patients with venous disorders of the lower limb. Eur J Vasc Endovasc Surg 2008;35:494-500. 